Disponível em

http://www.anpad.org.br/rac

RAC, Rio de Janeiro, v. 16, n. 3, art. 3, pp. 381-396, Maio/Jun. 2012

$(\mathrm{co}) \mathrm{EY}-\mathrm{NG}$

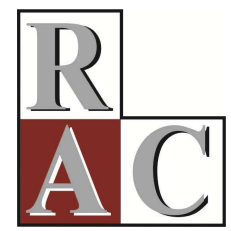

\title{
Características de Personalidade e Qualidade de Vida de Gestores no Rio Grande do Sul
}

\author{
Managers' Personality Characteristics and Quality of Life in Rio Grande do Sul
}

Simoni Missel D'Amico * E-mail: simoni@missel.com.br Universidade do Vale do Rio dos Sinos - UNISINOS

Porto Alegre, RS, Brasil.

Janine Kieling Monteiro

E-mail: janinekm@unisinos.br

Universidade do Vale do Rio dos Sinos - UNISINOS

Porto Alegre, RS, Brasil.

* Endereço: Simoni Missel D’Amico

Av.General Barreto Viana, 843, Bairro Chácara das Pedras, Porto Alegre/RS, 91330-630.

Copyright (C) 2012 RAC. Todos os direitos, até mesmo de tradução, são reservados. É permitido citar parte de artigos sem autorização prévia, desde que seja identificada a fonte. 


\title{
Resumo
}

O desenvolvimento da tecnologia e da globalização ocasionou transformações mundiais nas empresas, exigindo um novo perfil dos líderes organizacionais. Neste contexto, a personalidade tornou-se um fator determinante na obtenção do êxito profissional. A qualidade de vida (QV) é um tema de ampla aplicação em pesquisas. Através de uma pesquisa explicativa, buscou-se investigar que características de personalidade de gestores influenciam na sua qualidade de vida. No estudo da personalidade, foi utilizado o modelo dos Cinco Grandes Fatores. A amostra foi de 100 gestores de 27 empresas gaúchas, sendo 77\% homens e 23\% mulheres. Aplicou-se a Bateria Fatorial de Personalidade, o Instrumento da Organização Mundial de Saúde de QV - versão breve e um questionário sociodemográfico. Os resultados principais indicaram que há correlação negativa entre QV e o fator Neuroticismo, bem como associações positivas entre QV e o fator Extroversão, assim, indicando que ser mais comunicativo, ativo e gregário e ter menor instabilidade emocional são características associadas à saúde dos gestores. O Neuroticismo ainda demonstrou ser um fator preditivo da QV, apontando que gestores com maior ajustamento e estabilidade emocional têm maior bem-estar. Estes resultados podem auxiliar no planejamento de futuras intervenções visando à promoção da saúde destes indivíduos.

Palavras-chave: qualidade de vida; características de personalidade; gestor; liderança.

\begin{abstract}
Technology development and globalization caused worldwide changes in companies, requiring a new profile of organizational leaders. In this context, personality has become a decisive factor in obtaining professional success. Quality of life (QOL) is a broad research topic in management studies. By employing the Big Five Factors model, this explanatory research sought to investigate which personality characteristics of managers influence their quality of life. The sample consisted of 100 managers, with $77 \%$ men and $23 \%$ women, from 27 companies in Brazil's Rio Grande do Sul state. The study applied a Factorial Personality Test Battery, the brief version of the World Health Organization Quality of Life test (WHOQOL-BREF), and a socio demographic questionnaire. Main results indicated that there is a negative correlation between QOL and Neuroticism and positive associations between QOL and Extroversion, indicating that being more communicative, active, and gregarious and having less emotional instability are characteristics that positively affect managers' health. Furthermore, Neuroticism showed to be a predictor of QOL, demonstrating that better adjusted and more emotionally stable managers have greater wellbeing. These results may help the planning of future interventions aimed at promoting these individuals' health.
\end{abstract}

Key words: quality of life; personality characteristics; manager; leadership. 


\section{Introdução}

O desenvolvimento da tecnologia e da globalização favoreceu diversas fusões e aquisições de empresas que desencadearam transformações mundiais na estrutura, nos processos e no perfil dos profissionais que nelas trabalham. Uma consequência desta evolução é que a mesma requer desde a soma de novas habilidades até um novo perfil de líderes organizacionais (Wind, 1998), sendo que os líderes são, atualmente, peças fundamentais na gestão dos negócios (Sanchez \& Amendolara, 2008). O aumento da competição e a exigência, cada vez maior, de padrões elevados de qualificação e desempenho têm sido uma constante em todos os setores e situações de vida, esses fatores têm interferido na saúde das pessoas (Resende, 2000). Em relação às brutais transformações que vêm sofrendo o mundo corporativo, observa Kotter (1997), que as mudanças significativas e, frequentemente, traumáticas nas organizações têm crescido substancialmente. Diante destas questões, este estudo buscou investigar a relação entre características de personalidade e qualidade de vida em gestores.

Em nosso estudo, entende-se que os temas liderança e gestão são correlatos, pois todo gestor exerce um papel de influência ou de liderança na equipe que coordena. Gestão de pessoas remete ao uso de conhecimentos e técnicas necessárias para, através das pessoas, atingirem-se os objetivos da organização, ou seja, administrar, comandar, coordenar e controlar o grupo (Fayol, 1990). No Brasil, assim como no restante do mundo, nunca se falou tanto em liderança como nas últimas décadas, a razão disso é em função de que jamais, em qualquer época da história, essa habilidade foi tão necessária e valorizada (Vizioli \& Calegari, 2010). Impulsionadas pela globalização, empresas transnacionais, instaladas no Brasil, estão colaborando para a formação de um novo perfil profissional para gestores brasileiros. As mudanças que derivam desse cenário passam a exigir dos profissionais conhecimentos e aptidões diferenciadas para atuarem no ambiente atual de negócios (Echeveste, Vieira, Viana, Trez, \& Panosso, 1999). Na busca de gestores que apresentem esse novo perfil, as empresas veem-se sob uma nova pressão: recrutar, treinar e desenvolver líderes capazes de atender às demandas deste mercado e gerentes que deleguem mais (Handy, 1995).

\section{Características de personalidade}

A personalidade compreende uma construção pessoal, formada ao longo da vida, e tem seus alicerces no meio social que estamos inseridos, sendo também fruto de uma elaboração de nossa história de vida, isto é, da forma como sentimos, representamos e interagimos com as nossas experiências (Rubin \& Campbell, 1998). Um indivíduo pode agir e pensar diferentemente em situações diversas, dessa maneira, indicando que o comportamento pode ser variável de pessoa para pessoa, entretanto existem aspectos invariáveis no comportamento humano denominado de traço (Friedman \& Schustack, 2004). Assim, a personalidade pode ser definida como um processo dinâmico, que se encontra associado a um conjunto de traços que influenciam o funcionamento psicológico. Portanto o traço indica uma dimensão das diferenças individuais e padrões de pensamento, sentimentos e ações do indivíduo (McCrae \& John, 1992).

Diversos modelos teóricos têm contribuído para o estudo da personalidade, dentre eles, o modelo dos Cinco Grandes Fatores (CGF), conhecido, na literatura, como Big Five, ou Five Factor Model, o qual tem sido foco de interesse e entusiasmo crescentes por parte da comunidade científica. Esse modelo, além de ser "amplamente pesquisado, por representar uma forma de descrição da personalidade muito simples, elegante e econômica" (Nunes, Hutz, \& Nunes, 2008, p. 12), origina-se de um grande conjunto de pesquisas na área da personalidade. O modelo dos Cinco Grandes Fatores (CGF) é uma versão moderna da Teoria do Traço e um avanço conceitual e empírico no campo da personalidade. As teorias de traços de personalidade colaboraram grandemente para $\mathrm{o}$ desenvolvimento da sua base teórica e as teorias fatoriais contribuíram sob o aspecto instrumental e metodológico que convergiram para uma solução de cinco fatores, os quais descrevem dimensões humanas básicas de forma consistente (Nunes et al., 2008). Conforme Judge, Bono, Ilies e Gerhardt (2002), existe um consenso de que o modelo dos Cinco Grandes Fatores de personalidade pode ser 
utilizado para descrever os aspectos mais relevantes da personalidade e, por isto, esse modelo tem sido utilizado em pesquisas com diferentes grupos, incluindo os líderes.

Em nossa pesquisa, foi utilizada a Bateria Fatorial de Personalidade (BFP) - instrumento psicológico construído para a avaliação da personalidade a partir do modelo dos Cinco Grandes Fatores (CGF) - que inclui as dimensões Extroversão, Socialização, Realização, Neuroticismo e Abertura a Experiências. $\mathrm{O}$ fator Extroversão está relacionado às formas como as pessoas interagem entre si e indica o quanto elas são: comunicativas, ativas, assertivas, responsivas e gregárias. $\mathrm{O}$ fator Socialização descreve a qualidade e o tipo das relações interpessoais estabelecidas, que podem variar desde a compaixão e empatia até o antagonismo, ou seja, o quanto as pessoas se percebem capazes no convívio social (Nunes, Nunes, Cunha, Falcón, \& Saab, 2005). O fator Realização analisa características como grau de organização, persistência, controle, motivação e capacidade de planejamento de ações em função de uma meta (Nunes et al., 2008). O Neuroticismo tem sido considerado o fator mais associado às características emocionais das pessoas. Ele refere-se ao nível crônico de ajustamento e instabilidade emocional dos indivíduos e representa as diferenças individuais que ocorrem quando pessoas experienciam padrões emocionais associados a desconforto psicológico (aflição, angústia, sofrimento, etc.) e aos estilos cognitivos e comportamentais decorrentes (McCrae \& John, 1992).

Raros são os estudos encontrados que tratam sobre características de personalidade de gestores no contexto empresarial. Na revisão teórica sobre estudos que envolvem esta temática, optamos pela base de dados EBSCO, pois esta base indexa os principais periódicos dos diferentes continentes, assim, contemplado idiomas diversos, o que permite uma busca ampla. Em uma busca realizada nesta base de dados, então, utilizando os termos personality e manager, foram encontrados 59 resultados. No entanto, vários desses estudos não focalizam esses dois temas de forma mais ampla, mas enfocam apenas um desses dois aspectos. Como exemplos, podemos citar o estudo de Robson, Abraham e Weiner (2010) que examinou o potencial cognitivo e preditores de personalidade no trabalho de pessoas que prestam suporte e apoio direto a pessoas deficientes; e o de Garcia-Santos, Almeida, Werlang e Veloso (2010), o qual avaliou o processamento de informação de gestores de alto desempenho.

Destaca-se, entre os artigos encontrados, o estudo de Judge et al. (2002), o qual realizou uma meta-análise sobre as pesquisas que abordam liderança e traços de personalidade. Foi observado que algumas características como ajustamento, sociabilidade, integridade e autoconfiança aparecem relacionadas a traços de lideranças emergentes ou eficazes. Os autores procuraram analisar possíveis relações entre os traços de personalidade do modelo dos CGF e traços de liderança. Os resultados sugeriram que os fatores Extroversão, Realização e Abertura estão positivamente relacionados à liderança, enquanto que o fator Neuroticismo estaria negativamente associado a esse construto e que a relação entre Socialização (amabilidade) e liderança ainda mostra-se imprecisa. Outra pesquisa procurou investigar se as características de personalidade e comportamentais de gerentes de Tecnologia de Informação (TI) diferenciavam-se de gerentes de outros setores (Willcoxson \& Chatham, 2006). Essa demonstrou uma tendência nos Gerentes de TI em seguir instruções e prestar serviços, em vez de executar tarefas que demandem tomada de decisão e dúvidas quanto ao resultado esperado, ou seja, que estes gestores evitam correr riscos.

No contexto organizacional, verificou-se que alguns autores, como Mandelli (2001), Resende (2000) e Goleman (1995), destacam que a personalidade é mais importante e decisiva para o sucesso profissional das pessoas do que o conhecimento. Um programa de desenvolvimento pessoal que dê a cada um o conhecimento de suas próprias características de personalidade é de extrema importância e, indiscutivelmente, oportuno (Bergamini \& Tassinari, 2008). Os gestores e executivos deveriam reconhecer suas características de personalidade, pois o autoconhecimento é essencial para sobrevivência profissional (Drucker, 1995).

Pesquisas como a de Bruck e Allen (2003), sobre a relação entre a personalidade e o desempenho no trabalho, foram realizadas com base na teoria e na aplicação do modelo do CGF. Esse estudo teve como objetivo investigar as relações entre traços de personalidade e conflitos entre o 
trabalho e a família e os autores concluíram que as pessoas com níveis mais altos de realização reportaram menos interferência da família sobre o trabalho. Pessoas com níveis mais altos de Planejamento e Organização tendem a prevenir os conflitos com estes comportamentos. Níveis mais altos de Neuroticismo e de afeto negativo também estiveram associados com vários tipos de conflitos entre a família e o trabalho, evidenciando características de personalidade que envolvem a dificuldade de lidar com situações estressantes. Salgado (2002), por sua vez, investigou se os fatores como Extroversão, Realização e Neuroticismo eram preditores para o turnover. Não foram encontradas relações com o CGF e absenteísmo e acidentes de trabalho, no entanto os resultados apontaram que os fatores citados relacionam-se com a avaliação que os supervisores fazem do desempenho no trabalho e com o sucesso em programas de treinamento.

Outros estudos sobre personalidade também foram realizados examinando um amplo espectro de ocupações de profissionais liberais, como engenheiros, arquitetos, advogados e contadores, militares executivos, policiais, vendedores e profissionais com e sem qualificações, sendo identificado que algumas dimensões da personalidade interagem melhor em determinadas atividades do que em outras (Luminet, Bagby, Wagner, Taylor, \& Parker, 1999; Schinka, Dye, \& Curtiss, 1997).

\section{Qualidade de vida}

No contexto empresarial, a qualidade de vida é associada ao bem-estar dos funcionários, que inclui aspectos de saúde, ergonomia, segurança e lazer, o que, em pesquisa, traduz-se para Qualidade de Vida no Trabalho - QVT (Gonzaga, 2009). Nesse estudo, o termo Qualidade de Vida é alinhado ao conceito da Organização Mundial da Saúde de QV, que aborda as suas diferentes dimensões: a vida pessoal do indivíduo (social), seu ambiente de entorno (inclusive no trabalho), suas condições físicas e seu estado psicológico. Qualidade de Vida foi definida, pelo Grupo de Qualidade de Vida da Organização Mundial da Saúde, como "a percepção do indivíduo de sua posição na vida, no contexto da cultura e sistema de valores nos quais ele vive e em relação aos seus objetivos, expectativas, padrões e preocupações" (The WHOQOL Group, 1998, p. 1569).

A qualidade de vida inclui as condições de saúde do indivíduo e os aspectos do meio ambiente que podem ou não ser afetados pela saúde, como, por exemplo, a limitação no desempenho de papéis sociais ou a baixa performance nas atividades que desenvolve. As medidas de bem-estar referem-se a percepções subjetivas, relatos de sensações prazerosas ou desprazerosas e avaliações globais de saúde ou de estado subjetivo (Patrick \& Erickson, 1993). Ampliando, ainda, este conceito, a QV apóia-se na compreensão das necessidades humanas fundamentais, materiais e espirituais e tem, no conceito de promoção da saúde, seu foco mais relevante (Minayo, Hartz, \& Buss, 2000; Seidl \& Zannon, 2004).

O consenso quanto à multidimensionalidade do constructo refere-se ao reconhecimento de que o mesmo é composto por diferentes dimensões. A identificação dessas dimensões tem sido objeto de pesquisa científica em estudos empíricos, usando metodologias qualitativas estudadas por Bowling (1995) e quantitativas estudadas por Bowling (1995), Morris, Perez e McNoe (1998) e The World Health Organization Quality of Life Group (The WHOQOL Group, 1998). A natureza multidimensional do constructo foi validada de modo empírico a partir da emergência de quatro grandes dimensões ou fatores: (a) física, (b) psicológica, (c) do relacionamento social e (d) do ambiente. Além dessas dimensões, obteve-se uma avaliação da QV percebida de modo global, mensurada por quatro itens específicos que foram computados em um único escore. Segundo Seidl e Zannom (2004), existe uma tendência atual de usar demarcações focalizadas e combinadas para definir Qualidade de Vida, pois se acredita que estas podem contribuir para o avanço do conceito em bases científicas.

Visando a localizar pesquisas sobre o tema qualidade de vida e gestores, foi feita uma busca na base de dados EBSCO, com os descritores quality of life e manager, pela qual se obteve um resultado correspondente a 14 documentos, indicando uma escassez de estudos sobre esse assunto. Um dos estudos localizados foi o de Paiva e Couto (2008), o qual procurou descrever e analisar o corpo gerencial de uma empresa de Minas Gerais no que diz respeito às variáveis de QV no trabalho e 
estresse ocupacional, tendo em vista o contexto da reestruturação produtiva. $\mathrm{O}$ mesmo indicou que, de uma maneira geral, a QV dos gestores apresenta-se em níveis satisfatórios e que 58\% dos participantes consideraram que as fontes de pressão são elevadas, sugerindo uma realidade de estresse.

Outra pesquisa, realizada no Rio de Janeiro, analisou as inquietações e o bem-estar subjetivo em mulheres empreendedoras. Essa apontou que as empreendedoras estudadas compartilhavam de uma boa QV e mostravam-se destemidas e autoconfiantes, apesar de possuírem algumas dificuldades relativas à discriminação de gênero e à multiplicidade de papéis. Segunda a autora, a falta de medo e a autoconfiança, presentes nos discursos das empreendedoras, reproduzem características do perfil empreendedor observadas por inúmeros pesquisadores, independentemente de gênero ou de nacionalidade (Jonathan, 2005).

Os autores Lipp e Tangarelli (2007), Santos, Scandelari, Carvalho, Vaz e Santos (2009), Gonzaga (2009), Seidl e Zannon (2004) concordam que a QV se inter-relaciona com o equilíbrio em todas as áreas da vida (social, afetiva, saúde e profissional) e que as pessoas que se consideram felizes atribuem sua felicidade ao sucesso nas quatro áreas. $\mathrm{O}$ gestor pode estar permanentemente sujeito a situações geradoras de estresse no dia a dia do seu trabalho, como o não cumprimento de prazos, custos excessivos e baixa qualidade (do serviço prestado ou do produto) devido às falhas comuns que fogem de seu controle. Esses dados foram revelados através de um estudo com 100 gerentes. Destes, 45 gerentes apresentaram alto nível de estresse, 28 indicaram estresse leve ou moderado e apenas 27 foram individuados sem estresse. $\mathrm{O}$ estresse profissional prejudica a qualidade de vida dos indivíduos. Um dos fatores que causa estresse profissional está associado ao fato de que os resultados no trabalho dos gestores não dependem somente do seu desempenho pessoal, mas sim de seus subordinados, de seus pares, do contexto e, até mesmo, de seus superiores (Couto, 1987).

A partir de um estudo realizado em 10 países, Couto (1987) concluiu que os índices de estresse gerenciais mais elevados foram observados nos gestores do Egito e do Brasil. O diagnóstico de estresse, por meio do critério adotado, baseou-se na existência de instabilidade psíquica, com tendência à ansiedade, depressão e doenças psicossomáticas. Outros países pesquisados foram: Inglaterra, Suécia, Alemanha, Japão, Singapura, Estados Unidos, Nigéria e África do Sul. O autor verificou, ainda, que os índices mais baixos de estresse em gestores foram encontrados na Suécia e na Alemanha Ocidental. Destaca-se que a qualidade de vida dos gestores com elevados níveis de estresse fica prejudicada, considerando que as queixas deste grupo se referem à irritabilidade anormal diante de pequenas provocações, ou mesmo ocorrência de um comportamento agressivo sem ter sido provocado. Os gestores pesquisados referiram que se sentem como se estivessem sempre "com uma pedra na mão" e "com um pé atrás" no relacionamento com as pessoas.

No que diz respeito à associação entre características de personalidade e qualidade de vida, a grande maioria dos estudos encontrados está relacionada a grupos clínicos, tais como deprimidos, soropositivos, pacientes com câncer, etc. (Irigaray \& Schneider, 2009; Serafini \& Bandeira, 2009). Uma pesquisa desenvolvida com estudantes (Nunes, Hutz, \& Giacomoni, 2009) procurou investigar a relação entre bem-estar subjetivo e personalidade no modelo dos CGF. As facetas mais fortemente associadas à satisfação de vida foram depressão $(r=-0,56)$ e vulnerabilidade $(r=-0,35)$ e o fator foi Neuroticismo $(r=-0,49)$. Não foi encontrado nenhum estudo na base de dados EBSCO que buscasse relacionar esses dois constructos em gestores.

Tendo em vista a revisão apresentada, a qual aponta a escassez de estudos sobre a temática investigada, as mudanças exigidas no perfil dos gestores e a importância do seu papel na atualidade, a presente pesquisa teve como objetivo identificar a relação entre as características de personalidade de gestores e a qualidade de vida destes profissionais. Também foram investigados possíveis fatores preditores da qualidade de vida dos gestores. 


\section{Método de Pesquisa}

Esta pesquisa caracterizou-se como um estudo de caráter correlacional e explicativo, pois buscou conhecer as características de personalidade dos líderes que participaram desta amostra e relacioná-las com a sua qualidade de vida, bem como investigar fatores preditores da qualidade de vida no grupo em foco. Os dados obtidos foram analisados e interpretados no método quantitativo, por meio de dados numéricos.

\section{Amostra}

Fizeram parte da amostra desse estudo 100 gestores, homens (77\%) e mulheres (23\%), de 27 empresas situadas no Rio Grande do Sul. Como critério de inclusão foi utilizado o quesito trabalhar em empresas com, no mínimo, um ano de experiência em cargos de gestão. A seleção da amostra de líderes foi por conveniência e em parceria com os Recursos Humanos (RH) das empresas pesquisadas. Houve a participação de empresas de diversos segmentos como indústria, serviços e comércio, a maior parte delas do setor privado. A amostra caracterizou-se por líderes com idade entre 25 e 56 anos, com média de 38,77 anos e desvio padrão de 8,23. O tempo médio na empresa foi de 12,95 anos. Os gestores organizacionais que participaram deste estudo atuam no cargo de liderança, em média, há 8,36 anos e possuem, em média, 58 subordinados. O nível de instrução é, para a maior parte, ensino superior (73 participantes), sendo sete com ensino médio e 20 pós-graduados. Um elevado percentual dos pesquisados possui uma união estável (71\%) e tem de um a três filhos (67\%).

\section{Instrumentos de pesquisas}

Foram utilizados três instrumentos de pesquisa: uma ficha com dados sociodemográficos, o teste Bateria Fatorial de Personalidade - BFP (Nunes et al., 2008) - e o Questionário da Organização Mundial da Saúde sobre Qualidade de Vida - QV/OMS - Breve (Fleck, 2000). Na ficha de dados sociodemográficos investigaram-se informações referentes à idade, sexo, escolaridade, tempo de trabalho na empresa e no cargo de gestão, estado civil, número de filhos, área de atividade e número de pessoas sob a gestão do líder participante da pesquisa.

A BFP é composta por 126 itens de autoavaliação, que devem ser respondidos em uma escala likert que varia de 1 (absolutamente não me identifico com a frase) a 7 (descreve-me perfeitamente). $\mathrm{O}$ valor na escala aumenta quanto maior a identificação do sujeito com a questão. Ela identifica cinco fatores da personalidade (Extroversão, Socialização, Realização, Neuroticismo e Abertura para Novas Experiências, já anteriormente descritos) e, também, as facetas correspondentes a cada fator da personalidade. A consistência interna da escala apresentou alpha de Cronbach na razão de 0,89 para os fatores Neuroticismo e Extroversão, 0,85 para o de Socialização e 0,88 para os fatores de Realização e Abertura (Nunes et al., 2008).

Estudiosos enfatizam que a QV só pode ser avaliada pela própria pessoa, ao contrário das tendências iniciais de uso do conceito, quando a QV era avaliada por um observador, usualmente um profissional de saúde. Nesse sentido, o estudo de Slevin, Plant, Lynch, Drinkwater, e Gregory (1998) relata que há a preocupação quanto ao desenvolvimento de métodos de avaliação e de instrumentos que devem considerar a perspectiva da população ou dos pacientes, e não a visão de cientistas e de profissionais de saúde.

O Questionário da Organização Mundial da Saúde sobre Qualidade de Vida (QV/OMS) - Breve corresponde à versão em português do WHOQOL-bref, elaborada pelo Grupo de Qualidade de Vida da Organização Mundial de Saúde a partir do WHOQOL-100. A natureza multidimensional do constructo foi validada, de modo empírico, a partir de quatro grandes dimensões ou fatores: (a) física percepção do indivíduo sobre sua condição física; (b) psicológica - percepção do indivíduo sobre sua condição afetiva e cognitiva; (c) do relacionamento social - percepção do indivíduo sobre os relacionamentos sociais e os papéis sociais adotados na vida; (d) do ambiente - percepção do 
indivíduo sobre aspectos diversos relacionados ao ambiente onde vive (Seidl \& Zannon, 2004). O QV/OMS (versão breve) consta de 26 questões das quais duas questões são gerais de qualidade de vida e as demais representam cada uma das 24 facetas que compõem o instrumento original. Os dados que deram origem à versão abreviada foram extraídos do teste de campo de 20 centros, em 18 países diferentes. $\mathrm{O}$ instrumento apresenta características satisfatórias de consistência interna, validade discriminante, validade de critério, validade concorrente e fidedignidade teste-reteste (Fleck, 2000).

\section{Procedimentos de coleta e análise de dados}

A coleta de dados ocorreu no período entre julho e novembro de 2010 e teve início após o projeto ter sido aprovado pelo Comitê de Ética em Pesquisa da Universidade do Vale do Rio dos Sinos (Número da aprovação do CEP 10/067). Foram observados todos os procedimentos éticos referentes à pesquisa com seres humanos, incluindo a utilização do Termo de Consentimento Livre Esclarecido. O método utilizado para o processo de seleção dos gestores foi por conveniência e a participação foi voluntária. Foram marcadas reuniões para aplicação dos testes nas empresas através do RH ou dos gestores encarregados da organização da agenda. A aplicação dos instrumentos foi realizada de forma individual ou em grupo, no caso de haver mais de um gestor da mesma empresa participando deste estudo.

Os resultados dos instrumentos foram levantados e tabulados em um banco de dados, utilizando o SPSS for Windows (versão 18). A análise descritiva dos instrumentos envolveu medidas de tendência central, dispersão e propriedades da distribuição para as variáveis de interesse. Foi realizada uma correlação de Pearson a fim de avaliar a associação entre as características de personalidade e os fatores de QV. Posteriormente, foram realizadas análises de regressão linear múltipla para identificar o poder preditivo das características de personalidade em relação às dimensões de Qualidade de Vida e à QVtotal, adotando-se um nível de significância de 5\%. Foram feitas cinco análises de regressão múltipla pelo método stepwise, tendo, cada uma, como variável dependente uma das dimensões da QV: QVfísica, QVpsicológica, QVsocial, QVambiente e QVtotal e, como variáveis independentes, o conjunto de facetas da BFP. Nas análises de regressão, foi verificada a distribuição normal dos resíduos (através do Teste Kolmogorov-Smirnov), também, foi utilizado o teste de Durbin-Watson para considerar o diagnóstico de independência dos resíduos, assim, foram obtidos índices satisfatórios em todas as análises rodadas.

\section{Resultados e Discussões}

A apresentação dos resultados foi organizada em duas seções a fim de facilitar a compreensão das análises realizadas. Primeiramente, são apresentadas as análises descritivas dos testes utilizados, sendo conferidas as médias (m) e desvios padrão (DP) para cada uma das Características de Personalidade em seus fatores e facetas (Tabela 1) e para as subescalas de Qualidade de Vida (Tabela 2). A segunda seção trata das principais correlações observadas nas variáveis em estudo e, posteriormente, das análises de regressão empregadas.

\section{Análises descritivas das características de personalidade}

No nosso estudo, encontramos índices satisfatórios de consistência interna (alpha de Cronbach) da escala BFP, na razão de 0,87 para o fator Extroversão; 0,82 para o fator Realização; 0,75 para o fator Neuroticismo; 0,70 para o fator Abertura e 0,69 para o de Socialização.

$\mathrm{Na}$ amostra de líderes organizacionais que participou deste estudo $(\mathrm{n}=100)$, a dimensão de personalidade que apresentou o resultado mais alto corresponde ao fator denominado Socialização (m $=5,65$ e DP $=0,48$ ), conforme demonstrado na Tabela 1 . O fator Socialização descreve a qualidade e o tipo das relações interpessoais das pessoas e tem-se mostrado um fator importante no contexto do 
trabalho e das organizações (Nunes et al., 2005). Este resultado indica que os líderes estudados se avaliam com uma boa capacidade de convivência social, incluindo traços de empatia e compaixão pelo outro, o que sugere que dão importância às pessoas que estão sob sua coordenação. $O$ estudo internacional de meta-análise de pesquisas de Judge et al. (2002), já citado, concluiu que a relação entre o fator Socialização (amabilidade) e liderança ainda é imprecisa. No entanto percebe-se certa mudança no novo modelo gerencial no que diz respeito a uma perda gradual da importância dos chefes tradicionais, valorizados pelas competências técnicas, e o poder de influência, através da supervisão constante e coação, para dar lugar ao gestor líder voltado para o resgate da humanização no ambiente de trabalho, valorizando os sentimentos e a comunicação entre as pessoas (Araújo, 2001).

Tabela 1

\section{Características de Personalidade dos Líderes}

\begin{tabular}{llcc}
\hline \multicolumn{1}{c}{ Fatores } & \multicolumn{1}{c}{ Facetas } & Média & Desvio padrão \\
\hline Neuroticismo & & 2,55 & 0,56 \\
& Vulnerabilidade (N1) & 2,66 & 0,71 \\
& Instabilidade emocional (N2) & 2,84 & 1,07 \\
& Passividade (N3) & 3,09 & 0,94 \\
Extroversão & Depressão (N4) & 1,82 & 0,56 \\
& & 4,77 & 0,70 \\
& Comunicação (E1) & 4,75 & 1,03 \\
Socialização & Altivez (E2) & 3,90 & 0,84 \\
& Dinamismo (E3) & 5,52 & 0,70 \\
& Interações sociais (E4) & 5,12 & 0,96 \\
& & 5,65 & 0,48 \\
Realização & Amabilidade (S1) & 5,70 & 0,60 \\
& Pró-sociabilidade (S2) & 5,80 & 0,68 \\
& Confiança nas pessoas (S3) & 5,40 & 0,76 \\
Abertura & & 5,46 & 0,61 \\
& Competência (R1) & 5,80 & 0,59 \\
& Ponderação/prudência (R2) & 5,20 & 0,96 \\
& Empenho (R3) & 5,11 & 0,90 \\
& & 4,60 & 0,58 \\
& Interesse novas ideias (A1) & 4,44 & 0,88 \\
& & 4,59 & 0,68 \\
& & 4,86 & 0,89 \\
\hline
\end{tabular}

Nota. Fonte: Dados da Pesquisa.

$\mathrm{O}$ fator que apresentou o resultado mais baixo nesta amostra foi o Neuroticismo $(\mathrm{m}=2,55$; DP $=0,56$ ), que tem sido considerado o atributo mais associado às características emocionais das pessoas. Refere-se ao nível crônico de ajustamento e instabilidade emocional dos indivíduos (Costa \& Widiger, 2002) e representa as diferenças individuais que ocorrem quando pessoas experienciam padrões emocionais associados a desconforto psicológico (aflição, angústia, sofrimento, etc.) e os estilos cognitivos e comportamentais decorrentes. Níveis extremos de Neuroticismo, tanto muito elevados ou muito baixos, podem ser indicadores de padrões pouco adaptativos em relação aos seus componentes 
(McCrae \& John, 1992). Este dado aponta que os líderes estudados avaliam-se como sendo pessoas menos instáveis emocionalmente e que costumam ter um controle emocional em momentos de tensão. Esse resultado corrobora o estudo de Judge et al. (2002), o qual apontou que o fator Neuroticismo estaria negativamente relacionado a características de liderança.

\section{Características da qualidade de vida dos líderes}

$\mathrm{Na}$ nossa pesquisa, os índices de consistência interna (alpha de Cronbach), obtidos nas dimensões de Qualidade de Vida, foram: 0,65 para a QV física; 0,65 para a QV psicológica; 0,77 para a QV social e 0,69 para a QV ambiente.

O resultado do teste de Qualidade de Vida (QV/OMS Breve) indicou um índice mais favorável na dimensão Ambiente, como consta na Tabela 2. Os líderes participantes do estudo apresentaram uma pontuação mais elevada na dimensão Ambiente $(\mathrm{m}=31,22$; DP 3,91), referindo-se à segurança física e proteção, ambiente do lar, recursos financeiros, cuidados de saúde e sociais e oportunidades de adquirir novas informações e habilidades, ambiente físico e transporte. Os dados sugerem que os profissionais em cargos de gestão, nas organizações situadas no Rio Grande do Sul, estão satisfeitos com os ambientes em que vivem e trabalham e com a sua saúde física. O grau de instrução superior de 73\% dos profissionais estudados e uma melhor condição financeira, frequente em cargos de gestão, podem ter contribuído para os elevados índices nas dimensões Ambiente e Físico.

Tabela 2

\section{Qualidade de Vida - Domínios}

\begin{tabular}{lll}
\hline Domínios & Média & Desvio padrão \\
\hline Físico & 28,30 & 3,78 \\
Psicológico & 24,03 & 3,07 \\
Social & 11,91 & 2,18 \\
Ambiente & 31,22 & 3,91 \\
Total & 23,87 & 2,43 \\
\hline
\end{tabular}

Nota. Fonte: Dados da Pesquisa.

A menor média obtida no domínio social denota baixa qualidade de vida quanto às relações pessoais, suporte ou apoio social e atividade sexual. Embora os líderes desta amostra tenham avaliado que possuem maior satisfação com o ambiente em que vivem (conforto e bens materiais), demonstraram menor satisfação nas relações pessoais, familiares e sexuais. Possivelmente, eles possuem menos tempo para conviver com a família e com amigos fora do ambiente de trabalho em detrimento da dedicação à sua profissão, como indicam os resultados obtidos na pesquisa desenvolvida por Rabia e Christopoulos (2008). Estes autores buscaram analisar as causas de sofrimento no trabalho, por parte dos gestores, e a percepção destes sobre a integração de aspectos da qualidade de vida no trabalho e de sua vida pessoal. Como consequência do aumento da insegurança, da elevada carga de trabalho e dos constantes avanços da tecnologia, muitos gestores têm percebido perda de controle sobre seu trabalho. Um dado bastante relevante, no resultado da pesquisa realizada, é que 90\% dos entrevistados trabalham mais do que 40 horas semanais e, destes, $83 \%$ ultrapassam a 50 horas por semana, o que significa que há uma carga excessiva de trabalho em detrimento da vida pessoal, familiar e do lazer. Esta situação aumenta o estresse e vem afetando a saúde destes gestores. Os autores observaram que foi comum, entre os gestores entrevistados, o pensamento de que, para manter o emprego e salário, é necessário abrir mão do tempo para o lazer e família (Rabia \& Christopoulos, 2008). 


\section{Associações e análises de regressão entre características de personalidade e QV}

Considerando os objetivos deste estudo, foram analisadas possíveis associações entre características de personalidade e QV dos gestores pesquisados. Foi observado que quatro dos Cinco Grandes Fatores estão associados à QV, conforme a Tabela 3. O Neuroticismo e a Extroversão foram os fatores que mais fortemente se mostraram associados a diferentes dimensões de qualidade de vida abordadas neste estudo.

Tabela 3

Correlações entre QV e Características de Personalidade

\begin{tabular}{llccccc}
\hline & & NEURO & EXTRO & SOCIAL & REALIZ & ABERT \\
\hline QV Físico & Correl. Pearson & $-0,22^{*}$ & 0,19 & 0,045 & 0,11 & 0,07 \\
& Signif. (2-tailed) & 0,03 & 0,06 & 0,65 & 0,29 & 0,49 \\
QV Psicológico & Correl. Pearson & $-0,42^{* *}$ & $0,21^{*}$ & 0,12 & $0,21^{*}$ & $-0,04$ \\
& Signif. (2-tailed) & 0,00 & 0,03 & 0,25 & 0,03 & 0,67 \\
QV Social & Correl. Pearson & $-0,30^{* *}$ & 0,20 & $0,20^{*}$ & 0,10 & 0,06 \\
& Signif. (2-tailed) & 0,00 & 0,05 & 0,05 & 0,31 & 0,52 \\
\multirow{2}{*}{ QV Ambiente } & Correl. Pearson & $-0,34^{* *}$ & $0,25^{*}$ & $0,29 * *$ & $0,24^{*}$ & $-0,03$ \\
& Signif. (2-tailed) & 0,00 & 0,01 & 0,00 & 0,02 & 0,73 \\
QV Total & Correl. Pearson & $-0,42^{* *}$ & $0,29^{* *}$ & $0,22^{*}$ & $0,23^{*}$ & 0,01 \\
& Signif. (2-tailed) & 0,00 & 0,00 & 0,03 & 0,02 & 0,88 \\
\hline
\end{tabular}

Nota. Fonte: Dados da Pesquisa.

* A correlação é significativa ao nível de 0,05 , ** A correlação é significativa ao nível de 0,01 .

Os resultados indicaram correlações negativas significativas entre o fator Neuroticismo e todos os domínios de QV: QV Físico ( $\mathrm{r}=-0,22 ; \mathrm{p} \leq 0,03)$, QV Psicológico $(\mathrm{r}=-0,42 ; \mathrm{p}<0,01)$, QV Social $(\mathrm{r}=-0,29 ; \mathrm{p}<0,01)$, QV Ambiente $(\mathrm{r}=-0,34 ; \mathrm{p}<0,01)$ e QV Total $(\mathrm{r}=-0,42 ; \mathrm{p}<0,01)$, apontando que quanto menor o nível de Neuroticismo maior é a QV em todas as suas dimensões. Isto sugere que os gestores que apresentam maiores índices de qualidade de vida física, psicológica, de relações sociais e de meio ambiente têm maior estabilidade emocional e menor nível de aflição, angústia e sofrimento. Essa associação também foi encontrada no estudo de Nunes, Hutz e Giacomoni (2009), que investigou a relação entre bem-estar subjetivo e personalidade no modelo dos CGF em estudantes universitários, onde o fator Neuroticismo $(\mathrm{r}=-0,49)$ foi fortemente associado à satisfação de vida.

Existem, na literatura especializada, evidências significativas de que os pacientes deprimidos apresentam importante comprometimento da sua QV. A depressão é uma das facetas que compõe o fator Neuroticismo. Isso se deve, especialmente, ao fato de que os transtornos depressivos afetam vários domínios que fazem parte da avaliação global da QV. Sujeitos deprimidos apresentam déficits nas funções interpessoais, psicológicas, laborais e físicas (Kennedy, Eisfels, \& Cooke, 2001). Evidências recentes indicam que doenças afetivas estão associadas ao prejuízo na QV de seus portadores, sugerindo, ainda, que sujeitos acometidos por elas apresentam QV inferior àquela da população em geral (Goldney, Fischer, Wilson, \& Cheok, 2000). Os quadros de doenças afetivas ocasionam funcionamento social limitado em diversas áreas como no lazer, no trabalho, nas relações interpessoais, no estado de saúde e no desempenho acadêmico (Papakostas, Petersen, \& Mahal, 2004).

Foram observadas correlações positivas significativas entre Extroversão e QV Psicológico $(\mathrm{r}=$ $0,21 ; \mathrm{p} \leq 0,032)$, Extroversão e QV Social $(\mathrm{r}=0,19 ; \mathrm{p} \leq 0,050)$, Extroversão e QV Ambiente $(\mathrm{r}=0,25$; $\mathrm{p} \leq 0,012)$ e entre Extroversão e QV Total $(\mathrm{r}=0,28 ; \mathrm{p} \leq 0,004)$. Estes dados sugerem que os gestores mais dinâmicos e comunicativos, com mais facilidade de estabelecer interações sociais demonstram 
mais satisfação com a sua qualidade de vida. Os responsáveis pela área de recursos humanos enfrentam o desafio de exercer a liderança da transformação, dessa maneira, interagindo com funcionários de todos os níveis, transformando-os em agentes de mudança, conscientizando-os e habilitando-os para o ambiente de trabalho emergente (Moran, Harris, \& Stripp, 1997). Neste processo de mudança de postura profissional do gerente, a integração entre gestor e trabalhador pode viabilizar maior eficácia das inovações (Vidal, 1997).

Foi identificada correlação significativa $(r=0,21 ; p \leq 0,031)$ entre o fator Socialização e a QV Total, o que indica que quanto maiores são os níveis de Socialização (que se refere à capacidade do trabalhador seguir regras, ser persistente, voluntarioso, útil e cooperativo) maiores são os escores encontrados no QV Total dos líderes organizacionais. Ressalta-se novamente que o fator Socialização foi o que apresentou maior média entre os gestores, indicando ser a característica mais marcante no perfil dos participantes.

No que diz respeito à relação entre as facetas de cada um dos Cinco Grandes Fatores e a QV, as que foram mais fortemente associadas à QV total foram: o Empenho e Comprometimento $(\mathrm{r}=0,57$; $\mathrm{p}<0,001)$, a Depressão $(\mathrm{r}=-0,46 ; \mathrm{p}<0,001)$, a Confiança nas Pessoas $(\mathrm{r}=0,43 ; \mathrm{p}<0,001)$, o Dinamismo $(\mathrm{r}=0,39 ; \mathrm{p}<0,002)$ e a Instabilidade Emocional $(\mathrm{r}=-0,36 ; \mathrm{p}<0,001)$. O que indica que um alto nível de exigência pessoal atinente à qualidade das tarefas realizadas e dedicação às atividades profissionais com planejamento detalhado dos passos para a realização de alguma tarefa, dinamismo no trabalho e confiança nas pessoas são fatores que estão associados ao bem-estar. Bem como ter uma avaliação mais negativa dos eventos que ocorrem ao longo da vida está relacionada à menor QV total nos gestores estudados. Características estas, cada vez mais, valorizadas no perfil do gestor, o qual precisa alcançar metas, ser ágil e perspicaz para obter resultados superiores (Araújo, 2001; Silvius, 2009).

Como foi observada a associação de várias variáveis com a $\mathrm{QV}$, como última análise, foram realizadas cinco regressões lineares múltiplas, utilizando o método de stepwise, assim, intencionando verificar quais fatores poderiam ser preditores de QV total, tendo como variáveis independentes as dimensões do BFP e como dependente cada uma das dimensões de QV. Todos os modelos encontrados foram obtidos com um passo, tendo o fator Neuroticismo entrado como única variável preditora (Tabela 4). As dimensões QV psicológica $($ Beta $=-0,42)$ e $\mathrm{QV}$ total $($ Beta $=-0,42)$ foram aquelas em que o fator Neuroticismo obteve maior poder explicativo, explicando 17,8\% da propensão de ter menos QV psicológica e 18\% de ter menos QV total. Estes resultados corroboram a discussão anteriormente apresentada de que o Neuroticismo influenciou negativamente na Qualidade de Vida nos gestores estudados.

Tabela 4

Variáveis da BFP Preditoras das Dimensões de Qualidade de Vida

\begin{tabular}{lccccc}
\hline Variáveis & $\mathbf{R}$ & $\mathbf{R}^{\mathbf{2}}$ & Beta & $\mathbf{t}$ & $\mathbf{p}$ \\
\hline $\begin{array}{l}\text { QV Psicológica } \\
1 . \text { Neuroticismo }\end{array}$ & 0,42 & 0,178 & $-0,42$ & $-4,61$ & 0,000 \\
\hline $\begin{array}{l}\text { QV Física } \\
1 . \text { Neuroticismo }\end{array}$ & 0,22 & 0,490 & $-0,22$ & $-2,24$ & 0,000 \\
\hline $\begin{array}{l}\text { QV Ambiente } \\
1 . \text { Neuroticismo }\end{array}$ & 0,34 & 0,118 & $-0,34$ & $-3,62$ & 0,000 \\
\hline $\begin{array}{l}\text { QV Social } \\
1 . \text { Neuroticismo }\end{array}$ & 0,29 & 0,089 & $-0,29$ & $-3,63$ & 0,000 \\
\hline $\begin{array}{l}\text { QV total } \\
1 . \text { Neuroticismo }\end{array}$ & 0,42 & 0,180 & $-0,42$ & $-4,64$ & 0,000 \\
\hline
\end{tabular}

Nota. Fonte: Dados da Pesquisa. 


\section{Considerações Finais}

O objetivo principal deste estudo foi investigar possíveis associações entre características de personalidade e qualidade de vida dos profissionais analisados. Os fatores de personalidade que mais fortemente se mostraram correlacionados à qualidade de vida foram o Neuroticismo e a Extroversão. Indicando que ser mais comunicativo, ativo, responsivo e gregário e ter maior estabilidade emocional são características que influenciam positivamente na saúde e QV dos líderes estudados. Estes resultados sugerem que aspectos relacionados às habilidades sociais ainda são fatores essenciais nos líderes, os quais contribuem positivamente para a sua saúde. O Neuroticismo ainda demonstrou ser um fator preditivo da QV nesse grupo, indicando que gestores com maior ajustamento e estabilidade emocional têm maior saúde e bem-estar. Reforçando a importância de valorizar os aspectos e atributos emocionais dos gestores, como outros estudos já indicaram (Gonzaga, 2009).

A partir da análise de um grupo de gestores organizacionais quanto a características de personalidade e qualidade de vida, esta pesquisa pretende contribuir para o entendimento do ser humano em suas potencialidades e virtudes. Os resultados obtidos podem auxiliar no planejamento de futuras intervenções visando à promoção da saúde deste grupo. Denota-se a importância do gestor buscar um maior equilíbrio emocional no seu dia a dia, dessa forma, podendo auxiliar na sua QV, bem como na de seus subordinados, pois o gestor tem grande influência sobre o grupo. As empresas também deveriam se preocupar mais em investir no apoio emocional e na promoção de saúde dos seus gestores.

As limitações deste estudo referem-se a uma amostra de gestores não representativa das empresas do Rio Grande do Sul. Ressalta-se ainda que os dados, obtidos através dos instrumentos aplicados, são medidas de autorrelato e, por isso, descrevem a percepção pessoal do gestor em relação a si, podendo sofrer influências da desejabilidade social. Sugerem-se novas pesquisas formadas por amostras de profissionais de outras regiões do país, bem como em segmentos específicos, como área da saúde, empresas de serviços, indústria, dentre outras.

Artigo recebido em 14.06.2011 Aprovado em 12.03.2012.

\section{Referências}

Araújo, V. C. (2001). Gestão empresarial do século XXI: a mudança necessária. Administração em Revista, 1(1), 89-96.

Bergamini, C. W., \& Tassinari, R. (2008). Psicopatologia do comportamento organizacional. São Paulo: Cengage Learning.

Bowling, A. (1995). What things are important's in peoples lives? A survey of the publics judgements to inform scales of life related quality of life. Social Science \& Medicine, 41(10), 1447-1462.

Bruck, C. S., \& Allen, T. D. (2003). The relationship between big five personality traits, negative affectivity, type a behavior, and work-family conflict. Journal of Vocational Behavior, 63(3), 457-472. doi: 10.1016/S0001-8791(02)00040-4

Costa, P. T., Jr., \& Widiger, T. A. (2002). Introduction. In P. T. Costa Jr. \& T. A. Widiger (Eds.), Personality disorders and the five factor model of personality (2th ed., pp. 3-16) Washington, DC: American Psychological Association.

Couto, H. A. (1987). Stress e qualidade de vida. Rio de Janeiro: Cop Editora. 
Drucker, P. F. (1995). Administrando em tempos de grandes mudanças. São Paulo: Pioneira Thompson Learning.

Echeveste, S., Vieira, B., Viana, D., Trez, G., \& Panosso, C. (1999). O perfil do executivo no mercado globalizado. Revista da Administração Contemporânea, 3(2), 167-186. doi: 10.1590/S141565551999000200009

Fayol, H. (1990). Administração industrial e geral (9a ed.). São Paulo: Atlas.

Fleck, M. P. A. (2000). Aplicação da versão em português do instrumento abreviado de avaliação da qualidade de vida "WHOQOL-bref". Revista de Saúde Pública, 34(2), 178-183. doi: http://dx.doi.org/10.1590/S0034-89102000000200012

Friedman, H. S., \& Schustack, M. W. (2004). Teorias da personalidade: da teoria clássica à pesquisa moderna (B. Honorato, Trans.). São Paulo: Prentice Hall (Obra original publicada em 1999).

Garcia-Santos, S. C., Almeida, L. S., Werlang, B. S. G. \& Veloso, A. L. M. (2010). Processamento de informação em gestores de alto desempenho. Motricidade, 6(1), 85-102.

Goldney, R. D., Fischer L. J., Wilson, D. H., \& Cheok, F. (2000). Major depression and its associated morbidity and quality of life in a random, representative Australian community sample. Australian and New Zealand Journal of Psychiatry, 34(6), 1022-1029.

Goleman, D. (1995). Inteligência emocional, a teoria revolucionária que redefine o que é ser inteligente. Rio de Janeiro: Objetiva.

Gonzaga, A. R. (2009). Inteligência emocional e qualidade de vida em líderes organizacionais (Dissertação de mestrado não publicada). Universidade do Vale do Rio dos Sinos, São Leopoldo, RS, Brasil.

Handy, C. (1995). A era do paradoxo. São Paulo: Makron Books.

Irigaray, T. Q., \& Schneider, R. H. (2009). Dimensões de personalidade, qualidade de vida e depressão em idosas. Psicologia em Estudo, 14(4), 759-766. doi: 10.1590/S1413-73722009000400016

Jonathan, E. V. (2005). Mulheres empreendedoras: medos, conquistas e qualidade de vida. Psicologia em Estudo, 10(3), 373-382. doi: 10.1590/S1413-73722005000300005

Judge, T. A., Bono, J. E., Ilies, R., \& Gerhardt, M. W. (2002). Personality and leadership: a quantitative and qualitative review. Journal of Applied Psychology, 87(4), 765-785. doi: $10.1037 / / 0021-9010.87 .4 .765$

Kennedy, S. H., Eisfeld, B. S., \& Cooke, R. G. (2001). Quality of life: an important dimension in assessing the treatment of depression? Journal of Psychiatry and Neuroscience, 26(Suppl. S238), 23-28.

Kotter, J. (1997). Liderando mudança. São Paulo: Campus.

Lipp, M. E. N., \& Tangarelli, M. S. (2007). Stress e qualidade de vida em magistrados da justiça do trabalho: diferenças entre homens e mulheres. Psicologia: Reflexão e Crítica, 15(3), 537-548. doi: $10.1590 / \mathrm{S} 0102-79722002000300008$

Luminet, O., Bagby, R. M., Wagner H., Taylor, G. J., \& Parker, J. D. (1999). Relation between alexithymia and the five-factor model of personality: a facet-level analysis. Journal of Personality Assessment, 73(3), 345-358.

Mandelli, P. (2001). Muito além da hierarquia: revolucione sua performance como gestor de pessoas. São Paulo, SP: Gente. 
McCrae, R. R., \& John, O. P. (1992). An introduction to the five-factor model and its applications. Journal of Personality, 60(2), 175-215. doi: 10.1111/j.1467-6494.1992.tb00970.x

Minayo, M. C. S., Hartz, Z. M. A., \& Buss, P. M. (2000). Qualidade de vida e saúde: um debate necessário. Debate. Ciência \& Saúde Coletiva, 5(1), 7-18. doi: 10.1590/S141381232000000100002

Moran, R., Harris, P., \& Stripp, W. (1997). Desenvolvendo organizações globais: como preparar sua empresa para competição mundial. São Paulo: Futura.

Morris, J., Perez, D., \& McNoe, B. (1998). The use of quality of life data in clinical practice. Quality of Life Research, 7(1), 85-91.

Nunes, C H S., Hutz, C S., \& Giacomoni, C H. (2009). Associação entre bem estar subjetivo e personalidade no modelo dos cinco grandes fatores. Avaliação Psicológica, 8(1), 99-108.

Nunes, C. H. S. S., Hutz, C. S., \& Nunes, M. F. O. (2008). Manual técnico da bateria fatorial de personalidade - BFP. São Paulo: Casa do Psicólogo.

Nunes, C. H. S. S., Nunes, M. F. O., Cunha, T. F., Falcón, V., \& Saab, E. (2005, maio). Evidências da validade de critério da escala fatorial de socialização e extroversão: aplicação em um grupo de adictos a álcool ou outras substâncias. Resumo. Anais do Congresso do Instituto Brasileiro de Avaliação em Psicologia, Gramado, RS, Brasil, 3.

Paiva, K. C. M., \& Couto J. H. (2008). Qualidade de vida e estresse gerencial "pós-choque de gestão": o caso da Copasa - MG. Revista de Administração Pública, 42(6), 1189-1211. doi: $10.1590 /$ S0034-76122008000600008

Papakostas, G. I., Petersen, T., \& Mahal, Y. (2004). Quality of life assessments in major depressive disorder: a review of the literature. General Hospital Psychiatry, 26(1), 13-17. doi: 10.1016/j.genhosppsych.2003.07.004

Patrick, D. L., \& Erickson, P. (1993) Health status and health policy. Oxford: Oxford University Press.

Rabia, S., \& Christopoulos, T. P. (2008). Incompatibilidade entre vida pessoal e vida profissional dos gestores na era do conhecimento. Revista de Gestão USP, 15(3), 37-54.

Resende, E. (2000). O livro das competências. Rio de Janeiro: Qualitymark.

Robson, S. M., Abraham, J. D., \& Weiner, J. (2010). Characteristics of successful direct support professionals: an examination of personality and cognitive ability requirements. International Journal of Selection and Assessment, 18(2), 215-219. doi: 10.1111/j.1468-2389.2010.00503.x

Rubin, M. I., \& Campbell, T. J. (1998). The abc's of effective feedback: a guide for caring professionals. San Francisco, California: Jossey-Bass Publishers.

Salgado, J. F. (2002). The big five personality dimensions and counterproductive behaviours. International Journal of Selection and Assessment, 10(1/2), 117-125. doi: 10.1111/14682389.00198

Sanchez, A., \& Amendolara, L. (2008). O diretor executivo no direito brasileiro. Rio de Janeiro: Forense Universitário.

Santos, C. B., Scandelari, L., Carvalho, D. R., Vaz, M. S. M. G., \& Santos, M. G. P. (2009). Aquisição de conhecimento implícito de indicadores de qualidade de vida. Revista Brasileira de Qualidade de Vida, 1(1), 35-37. doi: 10.3895/S2175-08582009000100004 
Schinka, J. A., Dye, D. A., \& Curtiss, G. (1997). Correspondence between five-factor and RIASEC models of personality. Journal of Personality Assessment, 68(2), 355-368.

Seidl, E. M. F., \& Zannon, C. M. L. C. (2004). Qualidade de vida e saúde: aspectos conceituais e metodológicos. Caderno Saúde Pública, 20(2), 580-588. doi: 10.1590/S0102$311 X 2004000200027$

Serafini, A. J., \& Bandeira, D. R. (2009). Jovens vivendo com HIV/AIDS: a influência da rede de relações, do coping e do neuroticismo sobre a satisfação de vida. Revista de Psiquiatria do Rio Grande do Sul, 31(1), 51-59. doi: 10.1590/S0101-81082009000100010

Silvius, A. J. (2009). Project management 2027: the future of project management. In T. T. Kidd (Ed.), Handbook of research on technology project management, planning and operations (pp. 17-36). Pennsylvania, USA: Newtown Square.

Slevin, M. L., Plant, H. M., Lynch, D., Drinkwater, J., \& Gregory, W. M. (1998). Who should measure quality of life, the doctor or the patient? Br J Cancer, 57, 109-112.

The WHOQOL Group. (1998). The world health organization quality of life assessment (WHOQOL): development and general psychometric properties. Social Science \& Medicine, 46(12), 15691585 .

Vidal, E. M. (1997). O novo gerente para novas tecnologias: a visão de consultores de empresas. Revista Eletrônica da Administração, 3(2), 1-17. Recuperado em http://www.lume.ufrgs.br/handle/10183/19412

Vizioli, M., \& Calegari, M. L. (2010). Liderança: a força do temperamento. São Paulo: Pearson Prentice Hall.

Willcoxson, L., \& Chatham, R. (2006). Testing the accuracy of the IT stereotype: profiling IT managers'personality and behavioural characteristics. Information \& Management, 43(6), 697705. doi: 10.1016/j.im.2004.04.009

Wind, J. (1998, February). Towards a new marketing paradigm. Proceedings of the Winter Marketing Educadors Conference, Austin, Texas. 